\title{
Efficacy of levetiracetam combined with sodium valproate on pediatric epilepsy and its effect on serum miR-106b in children
}

\author{
JIAQIANG ZHAO, YAN SANG，YUAN ZHANG，DONGLI ZHANG，JIAO CHEN and XIAOMING LIU \\ Department of Neurology, Xuzhou Children's Hospital, Xuzhou Medical University, \\ Xuzhou, Jiangsu 221006, P.R. China
}

Received November 29, 2018; Accepted August 21, 2019

DOI: $10.3892 / e t m .2019 .8098$

\begin{abstract}
Efficacy of levetiracetam (LEV) combined with sodium valproate (SV) on pediatric epilepsy and its effect on serum miR-106b in children were investigated. One hundred and twenty children with epilepsy in Xuzhou Children's Hospital from July 2015 to July 2017 were enrolled, and divided into control group $(n=60)$ and observation group $(n=60)$ according to random sampling. Additionally, 100 children undergoing normal physical examination were collected as normal group. Patients in the control group were treated with SV, and patients in the observation group were treated with SV and LEV. RT-qPCR was used for detecting the relative expression of serum miR-106b in children. The clinical efficacy was evaluated. After treatment, the relative expression of serum miR-106b in the control group was significantly higher than that in the observation group $(\mathrm{P}<0.05)$. The difference in the control group was smaller than that in the observation group $(\mathrm{P}<0.05)$. According to the ROC curve analysis, when the cut-off value was 1.442 , the sensitivity, specificity and area under curve (AUC) of miR-106b in the diagnosis of pediatric epilepsy were $94.00,64.17$ and 0.833 respectively. The clinical efficacy in the observation group was significantly better than that in the control group $(\mathrm{P}<0.05)$. Spearman's test showed that the expression of miR-106b gradually decreased with the continuous improvement of the clinical efficacy $(\mathrm{P}<0.05)$. The AUC of miR-106b was $0.833,95 \%$ CI: 0.779 to 0.887 , the cut-off was 1.442 . LEV combined with SV is effective in the treatment of children with epilepsy, and does not increase the clinical ADR. The expression of serum miR-106b in children can be used as a clinical prognostic indicator and a potential diagnostic indicator.
\end{abstract}

Correspondence to: Dr Xiaoming Liu, Department of Neurology, Xuzhou Children's Hospital, Xuzhou Medical University, 18 Sudi North Road, Xuzhou, Jiangsu 221006, P.R. China

E-mail: minglx@yeah.net

Key words: levetiracetam, sodium valproate, pediatric epilepsy, miR-106b, efficacy

\section{Introduction}

Epilepsy is a chronic brain dysfunction syndrome caused by highly synchronous abnormal discharge of neurons in the brain (1). This common disorder in clinical neurology has sudden onset. Patients with epilepsy, mainly the elderly or children, are often accompanied by varying degrees of transient disturbance of consciousness, and patients with more severe epilepsy may have a general convulsion (2). It has been reported that (3) there are 50 patients diagnosed with epilepsy in 100,000 people in North America each year. Another study shows that (4) more than half of the 50 million patients with epilepsy in the world are in Asia. Childhood is a critical period for the development of human brain function, and the long-term abnormal discharge in the brain has a very adverse effect on the development of the brain function (5). The treatment of epilepsy in children needs to completely control the onset of the disease and eliminate the cause, as well as ensure the learning and daily living ability of children as much as possible. Therefore, how to actively and effectively treat children with epilepsy has become one of the problems that clinicians need to solve now.

New anti-epileptic drugs have emerged, bringing new hope for the treatment of epilepsy. The main component of levetiracetam (LEV) is a derivative of pyrrolidone. By binding to SV2A in the brain, synaptic vesicle protein $2 \mathrm{~A}$, LEV effectively inhibits the high-voltage-activated N-type $\mathrm{Ca}$ ion channel in the vertebral neurons of the hippocampus CA1 region, and thereby plays an anti-epileptic role (6). By improving the activity of glutamic acid decarboxylase, sodium valproate (SV) as a conventional therapeutic drug for epilepsy promotes the synthesis of $\gamma$-aminobutyric acid in the brain, inhibits $\gamma$-aminobutyrate transaminase and prevents the degradation of $\gamma$-aminobutyric acid, thereby improving the epileptic symptoms in patients (7).

MicroRNA (miR), a hot research topic in recent years, is a small (19-22 nucleotides) non-coding single-stranded RNA molecule. It causes the translational inhibition of target mRNA or the cleavage of mRNA by regulating gene expression (8). Studies in recent years have shown that miR is differentially expressed in tumor, cardiovascular and nervous system diseases (9-11). In the study by Sun et al (12), the expression of miR-106b is significantly increased in the serum of adult patients with epilepsy. However, there is no study showing 
the expression of miR-106b in pediatric epilepsy. Whether it can be a potential prognostic indicator has not been reported previously.

Therefore, the efficacy of LEV combined with SV in the treatment of pediatric epilepsy was analyzed in this study, and whether miR-106b could be used as a potential prognostic indicator for the treatment of children with epilepsy was also analyzed, in order to provide a reference for clinicians.

\section{Patients and methods}

A total of 120 children with epilepsy treated in Xuzhou Children's Hospital (Xuzhou, China) from July 2015 to July 2017 were enrolled, and divided into the control group $(n=60)$ and the observation group $(n=60)$ according to random sampling. The control group consisted of 31 males and 29 females, and the observation group consisted of 35 males and 25 females. Additionally, 100 children undergoing normal physical examination were collected as the normal group for this study.

This study was approved by the Ethics Committee of Xuzhou Children's Hospital, Xuzhou Medical University. Patients who participated in this research had complete clinical data. The family members of the children were informed of this study and signed an informed consent form.

Inclusion and exclusion criteria. Inclusion criteria: All children met the diagnostic criteria for pediatric epilepsy developed by The Basic and Clinical Aspects of Pediatric Neurological Diseases (13). Children had normal blood routine indicators, coagulation function and liver and kidney function.

Exclusion criteria: Children with a history of encephalitis, brain trauma and meningitis; children with immunodeficiency; children complicated with other malignant tumors; children treated with anti-epileptic drugs 1 month before enrollment; children with abnormalities in CT and MRI detection and children with an allergy to therapeutic drugs or a corresponding contraindication.

Drugs and source ofkits. SV (Hangzhou Sanofi Pharmaceutical Co., Ltd., SFDA approval number: H20010595), LEV (UCB Pharma S.A., approval number: H20160251), TransScript miRNA first-strand cDNA synthesis superMix and easypure miRNA kit (TransGen Biotech, AT351-01, ER601-01), PCR instrument (Applied Biosystems; Thermo Fisher Scientific, Inc., 7500). The primers were designed and synthesized by Sangon Biotech (Shanghai) Co., Ltd.

Treatment programs. Patients in the control group were treated with SV, and the specific treatment was as follows: the initial dose was $5-10 \mathrm{mg} /(\mathrm{kg} \mathrm{x}$ day). If epilepsy was not controlled 1 week after medication, the dose would be added with $5-10 \mathrm{mg} /(\mathrm{kg}$ x day) per week based on the initial dose, until the seizure did not occur [the maximum dose was controlled at $30-40 \mathrm{mg} /(\mathrm{kg} \times$ day $)$.

Patients in the observation group were treated with LEV and $\mathrm{SV}$, and the specific treatment was as follows: the initial dose was $10 \mathrm{mg} /(\mathrm{kg}$ x day), which was added once a week. The target dose was controlled at $20-60 \mathrm{mg} /(\mathrm{kg}$ x day) on the $3 \mathrm{rd}$ to 4 th week, the daily dose was divided into 2 doses.
$R T-q P C R$ detection. Venous blood $(5 \mathrm{ml})$ was collected from the child in the morning, allowed to stand at room temperature for $15-30 \mathrm{~min}$, and centrifuged at 3,000 x g for $10 \mathrm{~min}$ at $4^{\circ} \mathrm{C}$. Serum was collected and centrifuged at $10,080 \mathrm{x} \mathrm{g}$ for $10 \mathrm{~min}$ using an ultra-speed centrifuge at $4^{\circ} \mathrm{C}$ to remove the cell debris. A portion of the serum was taken for subsequent experiments, and the rest was stored at $-80^{\circ} \mathrm{C}$. The EasyPure miRNA kit was used for the extraction of total RNA from the serum, UV spectrophotometer and agarose gel electrophoresis for detecting the purity, concentration and integrity of the total RNA extracted, TransScript ${ }^{\circledR}$ miRNA RT Enzyme mix and 2X TS miRNA reaction mix (belonging to the PCR kit) for the reverse transcription of cDNA. The reverse transcription was carried out in strict accordance with the instructions, and cDNA was taken for subsequent experiments. PCR system was as follows: $1 \mu \mathrm{l}$ of cDNA, each of $0.4 \mu \mathrm{l}$ of upstream and downstream primers, $10 \mu \mathrm{l}$ of $2 \mathrm{X}$ TransStart ${ }^{\circledR}$ Top/Tip green qPCR supermix, passive reference dye (50X) (optional), and finally nuclease-free water supplemented to $20 \mu \mathrm{l}$. A two-step method was used for detection. PCR reaction conditions were: pre-denaturation at $94^{\circ} \mathrm{C}$ for $30 \mathrm{sec}$, denaturation at $94^{\circ} \mathrm{C}$ for $5 \mathrm{sec}$, annealing and extension at $60^{\circ} \mathrm{C}$ for $30 \mathrm{sec}$, for a total of 40 cycles. U6 was used as an internal reference gene in this experiment, and $2^{-\Delta \mathrm{Cq}}$ was used to express the relative expression of miR-106b. The primer sequences are shown in Table I (14).

Outcome measures. Main outcome measures: The clinical efficacy in the children was evaluated according to The Diagnosis and Curing Criteria of Clinical Diseases in 2002 (Table II). The difference in the expression of serum miR-106b was observed in children in the control group and the observation group before treatment and subjects in the normal group. The relative expression of serum miR-106b in children was observed before and 12 weeks after treatment, and that of serum miR-106b in children with different efficacy was observed.

Secondary outcome measures: The clinical data of children in the groups were observed. The ROC curve was plotted according to the expression of serum miR-106b in control, observation and normal group. The incidence of adverse drug reaction (ADR) was compared between the two groups.

Statistical analysis. In this study, SPSS20.0 software package was used for the statistical analysis of the data, GraphPad Prism 7 for plotting the figures. Count data were expressed as rate $(\%)$, tested by Chi-square and denoted by $\chi^{2}$. Measurement data were expressed as mean \pm standard deviation (means $\pm \mathrm{SD}$ ). Independent sample t-test was used for comparison between the groups, paired t-test for comparison in the group before and after treatment and denoted by $t$. Grade data were tested by rank sum test and denoted by $\mathrm{Z}$. Analysis of variance was used for comparison between multiple groups, LSD t-test for pairwise comparison in the group. The ROC curve was plotted according to the expression of serum miR-106b in the control, the observation and the normal group. The Spearman's test was used to analyze the relationship between the expression of miR-106b and the clinical efficacy. $\mathrm{P}<0.05$ was considered to indicate a statistically significant difference. 
Table I. Primer sequences.

\begin{tabular}{lcc}
\hline Genes & Upstream primer & Downstream primer \\
\hline miR-106b & 5'-TGCCTCCTCATTGTCTTCA-3' & 5'-GCCATCTCAAATACCTCCC-3' \\
U6 & 5'-CTCGCTTCGGCAGCACA-3' & 5'-AACGCTTCACGAATTTGCGT-3'
\end{tabular}

Table II. Clinical efficacy grading.

Efficacy grade Performance

\begin{tabular}{ll}
\hline Cured & No epileptic seizure in children \\
Markedly effective & No epileptic seizure or seizure frequency reduced by $>75 \%$ \\
Effective & Seizure frequency reduced by $25-75 \%$ \\
Invalid & Seizure frequency reduced by $<25 \%$, or no significant reduction, or increase in seizure
\end{tabular}

Table III. Clinical data of children.

\begin{tabular}{|c|c|c|c|c|c|}
\hline Factor & $\begin{array}{l}\text { Control group } \\
\qquad(\mathrm{n}=60)\end{array}$ & $\begin{array}{l}\text { Observation group } \\
\qquad(\mathrm{n}=60)\end{array}$ & $\begin{array}{l}\text { Normal group } \\
\qquad(\mathrm{n}=100)\end{array}$ & $\mathrm{t} / \chi^{2} / \mathrm{F}$ value & P-value \\
\hline \multicolumn{6}{|l|}{ Sex } \\
\hline Male & $31(51.67)$ & $35(58.33)$ & $58(58.00)$ & 0.742 & 0.690 \\
\hline Female & $29(48.33)$ & $25(41.67)$ & $42(42.00)$ & & \\
\hline Age (years) & $7.2 \pm 1.8$ & $7.5 \pm 1.9$ & $7.0 \pm 1.5$ & 1.624 & 0.200 \\
\hline $\operatorname{BMI}\left(\mathrm{kg} / \mathrm{m}^{2}\right)$ & $15.12 \pm 1.25$ & $14.82 \pm 1.42$ & $14.98 \pm 1.62$ & 0.623 & 0.537 \\
\hline Average course of disease (years) & $1.25 \pm 0.35$ & $1.28 \pm 0.29$ & & 0.511 & 0.610 \\
\hline Seizure frequency (times/years) & $2.48 \pm 1.22$ & $2.52 \pm 1.35$ & & 0.170 & 0.865 \\
\hline \multicolumn{6}{|l|}{ Epileptic seizure type } \\
\hline Simple partial seizure & $23(38.33)$ & $25(41.67)$ & & 1.110 & 0.893 \\
\hline Complex partial seizure & $11(18.33)$ & $9(15.00)$ & & & \\
\hline Tonic-clonic seizure & $10(16.67)$ & $11(18.33)$ & & & \\
\hline Tonic seizure & 7 (11.67) & $9(15.00)$ & & & \\
\hline Lennox-Gastaut syndrome & $9(15.00)$ & $6(10.00)$ & & & \\
\hline \multicolumn{6}{|l|}{ Place of residence } \\
\hline Urban & $35(58.33)$ & $30(50.00)$ & $58(58.00)$ & 1.170 & 0.557 \\
\hline Rural & 25 (41.67) & $30(50.00)$ & $42(42.00)$ & & \\
\hline
\end{tabular}

\section{Results}

Clinical data of children. The clinical data of children in the three groups were analyzed. There were no statistically significant differences in sex, age, BMI and place of residence between the three groups $(\mathrm{P}>0.05)$. The children in the control group and the observation group were compared. In the control group, the average course of disease was 1.25 years and the seizure frequency was 2.48 times/year; there were 23 children with simple partial seizure, 11 children with complex partial seizure, 10 children with tonic-clonic seizure, 7 children with tonic seizure and 9 children with Lennox-Gastaut syndrome. In the observation group, the average course of disease was 1.28 years and the seizure frequency was 2.52 times/year; there were 25 children with simple partial seizure, 9 children with complex partial seizure, 11 children with tonic-clonic seizure, 9 children with tonic seizure and 6 children with Lennox-Gastaut syndrome. There were no statistically significant differences in other data between the two groups (P>0.05) (Table III).

Relative expression of serum miR-106b in three groups of children. The expression of serum miR-106b in children before treatment was detected. Before treatment, the expression of serum miR-106b in the control group and the observation group was significantly higher than that in the normal group, with a significant difference $(\mathrm{P}<0.05)$; there was no statistically significant difference between the control group and the 
Table IV. Relative expression of miR-106b in two groups of children before and after treatment.

\begin{tabular}{lccrr}
\hline & \multicolumn{2}{c}{ Relative expression of miR-106b } & & \\
\cline { 2 - 3 } Group & Before treatment & After treatment & t value & P-value \\
\hline Control group (n=60) & $1.627 \pm 0.456$ & $1.454 \pm 0.302$ & 7.508 & $<0.001$ \\
Observation group (n=60) & $1.586 \pm 0.466$ & $1.244 \pm 0.256$ & 12.205 & $<0.001$ \\
t value & 0.487 & 4.109 & & \\
P-value & 0.627 & $<0.001$ & & \\
\hline
\end{tabular}

Table V. Comparison of difference in relative expression of miR-106b between the two groups before and after treatment.

\begin{tabular}{lcccr}
\hline Score & $\begin{array}{c}\text { Control group } \\
(\mathrm{n}=110)\end{array}$ & $\begin{array}{c}\text { Observation group } \\
(\mathrm{n}=110)\end{array}$ & $\mathrm{t}$ value & P-value \\
\hline Difference in relative expression of miR-106b & $0.172 \pm 0.128$ & $0.342 \pm 0.217$ & 5.227 & $<0.001$ \\
\hline
\end{tabular}

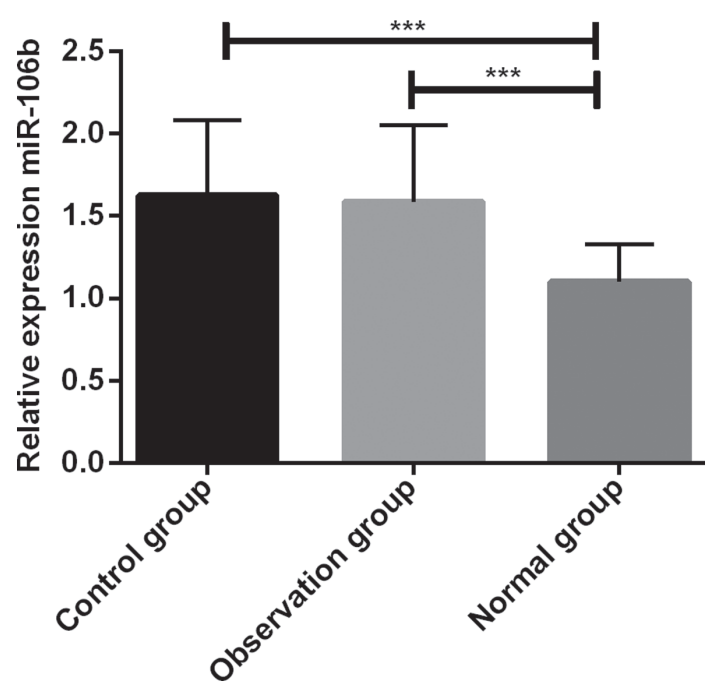

Figure 1. Relative expression of serum miR-106b in groups of children RT-qPCR showed that there was a difference in the expression of serum miR-106b in children between the groups. The expression of serum miR-106b in the normal group was significantly lower than that in the control group and the observation group $(\mathrm{P}<0.05)$. There was no significant difference between the control group and the observation group $(\mathrm{P}>0.05)$. ${ }^{* * *} \mathrm{P}<0.001$ indicates that the two groups were compared.

observation group $(\mathrm{P}>0.05)$ (Fig. 1). The expression of serum miR-106b in the two groups after treatment was significantly lower than that before treatment $(\mathrm{P}<0.05)$; after treatment, the relative expression of serum miR-106b in the control group was significantly higher than that in the observation group, with a statistically significant difference $(\mathrm{P}<0.05)$. The difference of miR-106b during the treatment was compared. The difference in the control group was smaller than that in the observation group, with a statistically significant difference $(\mathrm{P}<0.05)$ (Tables IV and V).

Diagnostic value of miR-106b in children with epilepsy. The serum miR-106b before treatment was detected in healthy children and in children with epilepsy. The area under curve (AUC)

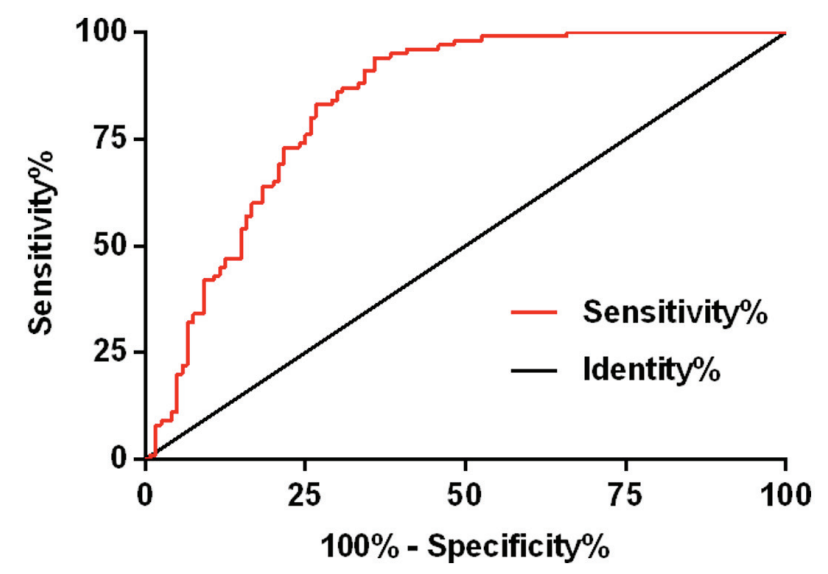

Figure 2. Diagnostic value of miR-106b in children with epilepsy. The ROC curve showed that the AUC of miR-106b was $0.833,95 \%$ CI: $0.779-0.887$ The maximum sensitivity and specificity obtained at 1.442 were $94.00 \%$ and $64.17 \%$, respectively. The Youden index was $58.17 \%$. AUC, area under curve.

of miR-106b was $0.833,95 \%$ CI: 0.779 to 0.887 the cut-off: 1.442. The sensitivity, the specificity and the Youden index were $94.00,64.17$, and $58.17 \%$, respectively (Fig. 2).

Relationship between clinical efficacy and miR-106b in two groups of children. The clinical efficacy in the two groups of children was compared. In the control group, there were 8 children cured, 20 children with marked effect, 18 children with effect and 14 children with invalidity. In the observation group, there were 15 children cured, 27 children with marked effect, 10 children with effect and 8 children with invalidity. The results of comparison showed that the clinical efficacy in the observation group was significantly better than that in the control group, with a statistically significant difference $(\mathrm{P}<0.05)$ (Table VI). The relationship between miR-106b and different clinical efficacy was analyzed. Spearman test showed that the expression of miR-106b gradually decreased with the continuous improvement of the clinical efficacy $(\mathrm{P}<0.05)$ (Table VII). 
Table VI. Clinical efficacy in two groups of children.

\begin{tabular}{lcccrrr}
\hline Groups & Cured & Markedly effective & Effective & Invalid & Z value & P-value \\
\hline Control $(\mathrm{n}=60)$ & $8(13.33)$ & $20(33.33)$ & $18(30.00)$ & $14(23.33)$ & -2.525 & 0.012 \\
Observation $(\mathrm{n}=60)$ & $15(25.00)$ & $27(45.00)$ & $10(16.67)$ & $8(13.33)$ & & \\
\hline
\end{tabular}

Table VII. Relationship between clinical efficacy and miR-106b.

\begin{tabular}{lcccr}
\hline Efficacy grade & $\mathrm{n}$ & Relative expression of miR-106b & r value & P-value \\
\hline Cured & 23 & $1.124 \pm 0.394$ & & $<0.001$ \\
Markedly effective & 47 & $1.319 \pm 0.284$ & 0.784 & \\
Effective & 28 & $1.239 \pm 0.298$ & & \\
Invalid & 22 & $1.658 \pm 0.207$ & & \\
\hline
\end{tabular}

Table VIII. ADR statistics.

\begin{tabular}{lcccccc}
\hline Groups & Nausea & Vomiting & Hypersomnia & Anorexia & Dizziness & Liver function damage \\
\hline Control $(\mathrm{n}=60)$ & $3(5.00)$ & $4(6.67)$ & $2(3.33)$ & $5(8.33)$ & $3(5.00)$ & $3(5.00)$ \\
Observation $(\mathrm{n}=60)$ & $2(3.33)$ & $3(5.00)$ & $2(3.33)$ & $3(5.00)$ & $2(3.33)$ & $2(3.33)$ \\
$\chi^{2}$ value & 0.209 & 0.152 & 0 & 0.536 & 0.209 & 0.209 \\
P-value & 0.648 & 0.697 & 1 & 0.464 & 0.648 & 0.648 \\
\hline
\end{tabular}

\begin{abstract}
ADR statistics. According to the ADR statistics, in the control group, there were 3 children with nausea, 4 children with vomiting, 2 children with hypersomnia, 5 children with anorexia, 3 children with dizziness and 3 children with liver function damage. In the observation group, there were 2 children with nausea, 3 children with vomiting, 2 children with hypersomnia, 3 children with anorexia, 2 children with dizziness and 2 children with liver function damage. The results of comparison showed that there was no statistically significant difference in the ADR between the two groups (P>0.05) (Table VIII).
\end{abstract}

\section{Discussion}

Epilepsy is a common syndrome in neurology (15). It has been reported that approximately 9 million people are affected by epilepsy in China, the population of which is mainly children and the elderly $(16,17)$. Compared with the elderly, the child's brain is at the developmental stage. Recurrent and transient tonic and clonic seizures for a long time easily cause hypoxia in the brain. Without timely intervention, the neurons of the child are damaged as the disease progresses, seriously affecting the intelligence and the daily life of the child. Therefore, the treatment of pediatric epilepsy is increasingly valued (18).

In this study, LEV and SV were used to treat children with epilepsy, and their clinical efficacy was observed. SV is the most commonly used broad-spectrum antiepileptic drug for the clinical treatment of epilepsy in clinical practice (19). Although the drug is effective, it is metabolized by the liver and may cause liver function damage in children. Besides, the antiepileptic treatment process is long and prone to various adverse reactions, which increases the treatment pain and reduces the treatment compliance of the child, resulting in unsatisfactory expected effect (20). Guo et al (21), reported that the long-term use of SV has a serious impact on growth and osteopenia in children with epilepsy. LEV is a new broad-spectrum antiepileptic drug for the treatment of epilepsy (22). There is a study showing that it has a lower effect on liver enzymes and creatine kinase in children (23). The clinical efficacy in the two groups of children after treatment was first observed. The improvement of the disease condition in the observation group was significantly better than that in the control group. In the study by Tan and Appleton (24), 26 children with epilepsy aged under 10 years were treated with LEV alone, and $61 \%$ of them had a good response to LEV, with the seizure frequency reduced by at least $50 \%$. In addition, 2 children with refractory epilepsy had no seizure after medication. In this study, the effective rate of LEV combined with SV for the treatment of children was $93.64 \%$, and the efficacy was significantly better than that of single medication. This suggests that the combined medication can effectively improve the condition of the child. Then, the adverse events during the treatment were compared between the two groups. There was no difference in the ADR between the two groups. This suggests that LEV and SV have good efficacy in the treatment of children with epilepsy, and have small adverse reactions.

miR-106b is an important member of the miR-17 family and differentially expressed in tumor and cardiovascular 
diseases $(25,26)$. According to Cava et al (27), highly expressed in the serum of patients with epilepsy, miR-106b is expected to become a new diagnostic indicator. However, there is no relevant research on whether it can be a prognostic indicator in the treatment of epilepsy. Therefore, in this study, whether miR-106b can be used as a prognostic indicator in the treatment of children with epilepsy was investigated. The expression of serum miR-106b in children with epilepsy was first detected. The expression of serum miR-106b in the control group and the observation group was significantly higher than that in the normal group, consistent with the results of Pitkänen et al (28). Subsequently, the ROC curve was plotted. In the study by An et al (29), the AUC of miR-106b was 0.786, 95\% CI: 0.693-0.824, while the AUC was $0.882,95 \%$ CI: $0.839-0.926$ in the study by Wang et al (30), consistent with the results of this study. In the present study, the AUC of miR-106b was 0.833 , 95\% CI: 0.779-0.887. These findings suggest that miR-106b can be used as a potential diagnostic indicator for children with epilepsy. Finally, the correlation analysis between the clinical efficacy and the expression of miR-106b in children was performed. The expression of miR-106b in children after treatment gradually decreased with the improvement of the disease, suggesting that miR$106 \mathrm{~b}$ is expected to be a potential prognostic indicator for children with epilepsy after treatment.

This study showed the clinical efficacy of LEV combined with SV in children with epilepsy. The combination of the two drugs effectively improves the condition of the child, and does not increase the clinical ADR. The expression of serum miR-106b in children was detected. miR-106b was highly expressed in children with epilepsy. The expression of serum miR-106b in children can be used as a prognostic indicator. However, there are still limitations in this study. First, the children were not followed up for a long time, and the specific improvement of them remains unclear. Secondly, the specific mechanism of LEV combined with SV on improving of the expression of miR-106b in children needs further investigation.

In summary, LEV combined with $\mathrm{SV}$ is effective in the treatment of children with epilepsy, and does not increase the clinical ADR. The expression of serum miR-106b in children can be used as a clinical prognostic indicator and a potential diagnostic indicator.

\section{Acknowledgements}

Not applicable.

\section{Funding}

No funding was received.

\section{Availability of data and materials}

The datasets used and/or analyzed during the present study are available from the corresponding author on reasonable request.

\section{Authors' contributions}

JZ wrote the manuscript. JZ and YS were responsible for PCR. YZ and DZ assisted with the clinical efficacy analysis. JC and
XL were responsible for the statistical analysis. All the authors read and approved the final manuscript.

\section{Ethics approval and consent to participate}

The study was approved by the Ethics Committee of Xuzhou Children's Hospital, Xuzhou Medical University (Xuzhou, China). Patients who participated in this research, had complete clinical data. The family members of the children were informed of this study and signed an informed consent form.

\section{Patient consent for publication}

Not applicable.

\section{Competing interests}

The authors declare that they have no competing interests.

\section{References}

1. Berg AT and Scheffer IE: New concepts in classification of the epilepsies: Entering the 21st century. Epilepsia 52: 1058-1062, 2011.

2. Russ SA, Larson $\mathrm{K}$ and Halfon N: A national profile of childhood epilepsy and seizure disorder. Pediatrics 129: 256-264, 2012.

3. Theodore WH, Spencer SS, Wiebe S, Langfitt JT, Ali A, Shafer PO, Berg AT and Vickrey BG: Epilepsy in North America: A report prepared under the auspices of the global campaign against epilepsy, the International Bureau for Epilepsy, the International League Against Epilepsy, and the World Health Organization. Epilepsia 47: 1700-1722, 2006.

4. Mac TL, Tran DS, Quet F, Odermatt P, Preux PM and Tan CT: Epidemiology, aetiology, and clinical management of epilepsy in Asia: A systematic review. Lancet Neurol 6: 533-543, 2007.

5. Glennon JM, Weiss-Croft L, Harrison S, Cross JH, Boyd SG and Baldeweg T: Interictal epileptiform discharges have an independent association with cognitive impairment in children with lesional epilepsy. Epilepsia 57: 1436-1442, 2016.

6. Belcastro V, Costa C, Galletti F, Autuori A, Pierguidi L, Pisani F, Calabresi P and Parnetti L: Levetiracetam in newly diagnosed late-onset post-stroke seizures: A prospective observational study. Epilepsy Res 82: 223-226, 2008.

7. Chen L, Feng P, Wang J, Liu L and Zhou D: Intravenous sodium valproate in mainland China for the treatment of diazepam refractory convulsive status epilepticus. J Clin Neurosci 16: 524-526, 2009.

8. Ha M and Kim VN: Regulation of microRNA biogenesis. Nat Rev Mol Cell Biol 15: 509-524, 2014.

9. Condorelli G, Latronico MV and Cavarretta E: microRNAs in cardiovascular diseases: Current knowledge and the road ahead. J Am Coll Cardiol 63: 2177-2187, 2014.

10. Lin S and Gregory RI: MicroRNA biogenesis pathways in cancer. Nat Rev Cancer 15: 321-333, 2015.

11. Sun Y, Luo ZM, Guo XM, Su DF and Liu X: An updated role of microRNA-124 in central nervous system disorders: A review. Front Cell Neurosci 9: 193, 2015.

12. Sun J, Cheng W, Liu L, Tao S, Xia Z, Qi L and Huang M: Identification of serum miRNAs differentially expressed in human epilepsy at seizure onset and post-seizure. Mol Med Rep 14: 5318-5324, 2016.

13. Healy F and Panitch HB: Pulmonary complications of pediatric neurological diseases. Pediatr Ann 39: 216-224, 2010.

14. Livak KJ and Schmittgen TD: Analysis of relative geneexpression data using real-time quantitative PCR and the 2(-Delta Delta C(T)) method. Methods 25: 402-408, 2001

15. Fisher RS, Acevedo C, Arzimanoglou A, Bogacz A, Cross JH, Elger CE, Engel J Jr, Forsgren L, French JA, Glynn M, et al: ILAE official report: A practical clinical definition of epilepsy. Epilepsia 55: 475-482, 2014.

16. Singh A and Trevick S: The epidemiology of global epilepsy. Neurol Clin 34: 837-847, 2016. 
17. Ollenberger GP, Byrne AJ, Berlangieri SU, Rowe CC Pathmaraj K, Reutens DC, Berkovic SF, Scheffer IE and Scott AM: Assessment of the role of FDG PET in the diagnosis and management of children with refractory epilepsy. Eur J Nucl Med Mol Imaging 32: 1311-1316, 2005.

18. Lagunju IA, Oyinlade AO and Babatunde OD: Seizure-related injuries in children and adolescents with epilepsy. Epilepsy Behav 54: 131-134, 2016.

19. Stephen LJ, Kwan P, Shapiro D, Dominiczak M and Brodie MJ: Hormone profiles in young adults with epilepsy treated with sodium valproate or lamotrigine monotherapy. Epilepsia 42: 1002-1006, 2001.

20. Fisher K, Vuppalanchi R and Saxena R: Drug-induced liver injury. Arch Pathol Lab Med 139: 876-887, 2015.

21. Guo CY, Ronen GM and Atkinson SA: Long-term valproate and lamotrigine treatment may be a marker for reduced growth and bone mass in children with epilepsy. Epilepsia 42: 1141-1147, 2001.

22. Weijenberg A, Brouwer OF and Callenbach PM: Levetiracetam monotherapy in children with epilepsy: A systematic review. CNS Drugs 29: 371-382, 2015.

23. Attilakos A, Dinopoulos A, Paschalidou M, Tsirouda M, Prasouli A, Siafakas N and Garoufi A: Effect of levetiracetam monotherapy on liver enzymes and creatine kinase concentrations in children with epilepsy: A prospective study. J Clin Neurol 14: 594-595, 2018.

24. Tan MJ and Appleton RE: Efficacy and tolerability of levetiracetam in children aged 10 years and younger: A clinical experience. Seizure 13: 142-145, 2004.
25. Moshiri F, Salvi A, Gramantieri L, Sangiovanni A, Guerriero P, De Petro G, Bassi C, Lupini L, Sattari A, Cheung D, et al: Circulating miR-106b-3p, miR-101-3p and miR-1246 as diagnostic biomarkers of hepatocellular carcinoma. Oncotarget 9: 1535015364, 2018.

26. Wronska A, Kurkowska-Jastrzebska I and Santulli G: Application of microRNAs in diagnosis and treatment of cardiovascular disease. Acta Physiol (Oxf) 213: 60-83, 2015.

27. Cava C, Manna I, Gambardella A, Bertoli G and Castiglioni I: Potential role of miRNAs as theranostic biomarkers of epilepsy. Mol Ther Nucleic Acids 13: 275-290, 2018.

28. Pitkänen A, Löscher W, Vezzani A, Becker AJ, Simonato M, Lukasiuk K, Gröhn O, Bankstahl JP,Friedman A, Aronica E, et al: Advances in the development of biomarkers for epilepsy. Lancet Neurol 15: 843-856, 2016.

29. An N, Zhao W, Liu Y, Yang X and Chen P: Elevated serum miR-106b and miR-146a in patients with focal and generalized epilepsy. Epilepsy Res 127: 311-316, 2016.

30. Wang J, Yu JT, Tan L, Tian Y, Ma J, Tan CC, Wang HF, Liu Y, Tan MS, Jiang T, et al: Genome-wide circulating microRNA expression profiling indicates biomarkers for epilepsy. Sci Rep 5: 9522,2015

c) (i) $\ominus$ This work is licensed under a Creative Commons

Attribution-NonCommercial-NoDerivatives 4.0 International (CC BY-NC-ND 4.0) License. 